\title{
Aplicação do modelo HidroBacia na microbacia do córrego Jaqueira, Espírito Santo
}

\section{Application of hydrological model HidroBacia in Jaqueira stream watershed, Espírito Santo}

\author{
Leonardo Nazário Silva dos Santos ${ }^{1}$; Roberto Avelino Cecílio ${ }^{2 *}$; Sidney Sara Zanetti ${ }^{3}$
}

\section{Resumo}

A modelagem hidrológica aplicada em bacias hidrográficas consiste numa das principais ferramentas para a gestão dos recursos hídricos, visando minimizar o processo de degradação ambiental. O presente trabalho objetivou avaliar a aplicabilidade do modelo hidrológico HidroBacia em uma microbacia hidrográfica de Alegre-ES, além de compará-lo com os métodos Racional (MR) e Número da Curva (MNC). O processo de infiltração de água no solo no HidroBacia, representado pela equação de GreenAmpt-Mein-Larson (GAML), foi simulado por meio de 72 diferentes combinações de parâmetros de entrada, a fim de evidenciar a de melhor desempenho. Compararam-se dados reais (obtidos no exutório da microbacia) de vazão máxima de escoamento superficial $\left(Q_{\operatorname{máx}}\right)$ e lâmina escoada (LES) com aqueles simulados pelo HidroBacia e pelos MR e MNC. Verificou-se que sete, das 72 combinações, melhor estimaram $\mathrm{Q}_{\text {máx }}$ e LES nas simulações realizadas com o HidroBacia. Os dados simulados pelo MR e MNC superestimaram a $Q_{\text {máx }}$ e a LES, respectivamente.

Palavras-chave: Escoamento superficial, equação de Green-Ampt-Mein-Larson, infiltração

\begin{abstract}
Hydrologic simulation of watersheds is an useful tool for the water resources management and to reduce environmental degradation in watersheds. This work evaluated the applicability of the hydrologic modeling in a small watershed using the HidroBacia model at Alegre-ES and compare the results of flow maximum $\left(\mathrm{Q}_{\text {max }}\right)$ and surface runoff flow (LES) in watershed "outlet" with rational methods (MR) and Curve Number (MNC). The soil water infiltration process is represented by means of the Green-Ampt equation, modified by Mein and Larson (GAML) in this model. This equation needs the parameters: matric potential in the wetting front, hydraulic conductivity and soil moisture in the "field saturation". This work assessed seventy two input combinations of parameters for equation GAML. Compared to real data (obtained at the watershed "outlet") the $\mathrm{Q}_{\text {max }}$ and LES with the simulated by HidroBacia and by the MR and MNC. Verify in this work seven best combinations estimated $\mathrm{Q}_{\text {máx }}$ and LES in simulations with HidroBacia. The simulated data by the MR and MNC overestimated $\mathrm{Q}_{\text {máx }}$ and LES, respectively. Therefore, for experimental condition, recommend the use of hydrological model HidroBacia to estimate both parameters the $\mathrm{Q}_{\text {max }}$ and the LES.
\end{abstract}

Key words: Runoff, equation of Green-Ampt-Mein-Larson, infiltration

\footnotetext{
${ }^{1}$ Eng $^{\mathrm{o}} \mathrm{Agr}^{\circ}$, Discente de Doutorado em Engenharia Agrícola, Faculdade de Engenharia Agrícola, Universidade Estadual de Campinas, UNICAMP, Campinas, SP. Bolsista FAPESP Processo nº 2010/15382-8. E-mail: nazarioss@yahoo.com.br

${ }^{2}$ Eng $^{\circ}$ Agrícola, Prof. Dr. do Dept ${ }^{\circ}$ de Ciências Florestais e da Madeira, Universidade Federal do Espírito Santo, UFES. Alegre, ES. Bolsista de Produtividade em Pesquisa do CNPq. E-mail: roberto.cecilio@ufes.br

${ }^{3}$ Eng $^{\circ}$ Agr $^{\circ}$, Prof. Dr. do Dept ${ }^{\circ}$ de Ciências Florestais e da Madeira, UFES. Alegre, ES. E-mail: sidney.zanetti@ufes.br

* Autor para correspondência
} 


\section{Introdução}

Dentre as fases do ciclo hidrológico, o escoamento superficial é aquela que corresponde ao deslocamento de água sobre a superfície do solo (SILVA et al., 2006a; CARLESSO et al., 2011). No entanto, sua ocorrência pode gerar consequências como: enchentes, redução das vazões nos cursos d'água no período de estiagem, erosão acelerada do solo, assoreamento dos recursos hídricos (BERTOL et al., 2007; PINTO et al., 2009; SILVA et al., 2009b), poluição e eutrofização das águas devido ao transporte de fertilizantes, nutrientes, matéria orgânica, sementes e defensivos agrícolas (OLIVEIRA et al., 2010); ou seja, potencializa a degradação ambiental e resulta em prejuízos diretos à produção agrícola e pecuária. Nesse sentido, seu estudo é fundamental para o adequado planejamento e manejo de bacias hidrográficas visando à sustentabilidade do agronegócio (LOURENÇÃO; HONDA, 2007), bem como, o dimensionamento de obras hidráulicas em bacias urbanas e rurais (SILVA et al., 2006b; BERTOL et al., 2008).

Diante disto, a utilização de modelos capazes de prever o comportamento hidrológico de bacias hidrográficas em função de modificações no uso da terra, se tornou ferramenta indispensável para o planejamento e a sustentabilidade. Entretanto, percebe-se que alguns modelos possuem limitações, principalmente por não considerarem os diversos fatores que influenciam na produção de escoamento superficial e, consequentemente, na ocorrência de erosão hídrica, tais como a variabilidade espacial das propriedades físico-hídricas do solo e da cobertura de superfície. Isto pode se agravar, quando se deseja avaliar determinadas regiões tropicais, pois, segundo Zanetti et al. (2009a), os modelos desenvolvidos no exterior apresentam limitações quanto ao seu uso para as condições edafoclimáticas brasileiras. Neste contexto, a aplicabilidade e o ajuste dos parâmetros desta ferramenta têm sido um desafio para os profissionais e pesquisadores quando se avalia a realidade brasileira. Estes modelos são de difícil implantação para o manejo de uso de solo e água em bacias hidrográficas, devido à sua complexidade e dinâmica ambiental, no tempo e no espaço (CAO; VERVOORT; DABNEY, 2011).

Dentre os modelos existentes para estimar o escoamento superficial, os mais utilizados são o Método Racional (MR) e o Método do Número da Curva (MNC) (SAHU; MISHRA; ELDHO, 2010; ROSATTO et al., 2011), pois são simples e, de fácil aplicação (SILVA et al., 2009a; JABUR; RIZZI, 2011). Outros modelos foram desenvolvidos baseando-se no ciclo hidrológico, o que os torna aplicáveis em quaisquer condições edafoclimáticas. Silva et al. (2009b) e Zanetti et al. (2009a) desenvolveram um modelo hidrológico aplicável por intermédio de software, denominado HidroBacia, que permite a realização do balanço hídrico, a obtenção do hidrograma de escoamento superficial, da vazão máxima e do volume escoado para qualquer ponto de uma bacia hidrográfica. Entretanto, os estudos com o modelo HidroBacia são recentes, tornando-se necessários estudos consistentes sobre seu comportamento, além da realização de estudos sobre a avaliação de seu desempenho em outras bacias de drenagem.

Do exposto, o presente trabalho objetivou avaliar a aplicabilidade do modelo hidrológico HidroBacia para estimar a vazão máxima e a lâmina de escoamento superficial na microbacia hidrográfica do córrego Jaqueira, no Espírito Santo e, comparar as estimativas com as obtidas pelos métodos Racional e Número da Curva.

\section{Material e Métodos}

\section{Localização e caracterização da área de estudo}

O trabalho foi desenvolvido na microbacia hidrográfica do córrego Jaqueira, com área de 21,6 ha, pertencente à bacia do rio Itapemirim, no município de Alegre-ES, com as seguintes coordenadas geográficas: latitude máxima igual a $-20^{\circ} 45^{\prime} 16,2$ " e mínima igual a $-20^{\circ} 45^{\prime} 36,72$ "; longitude máxima de $-41^{\circ} 31^{\prime} 44,76^{\prime \prime}$ e mínima de $-41^{\circ} 31^{\prime} 25,32^{\prime \prime}$. 
Para a caracterização da área, foi realizado um levantamento planialtimétrico da microbacia e áreas de entorno utilizando o equipamento GPS geodésico estático (modelo GTR-A da TechGeo®). Após o levantamento de campo, realizou-se a interpolação das cotas para a obtenção do modelo de elevação digital do terreno - MDE (Figura 1a), sendo utilizados pixels com dimensões de $3 \times 3$ metros, a partir do programa TOPOGRID no ArcInfo Workstation. Em seguida, usando o ArcView GIS® 3.2a, delimitou-se a bacia hidrográfica do córrego Jaqueira.

Figura 1. Microbacia hidrográfica do córrego Jaqueira: modelo digital de elevação (a) e uso e ocupação do solo (b).

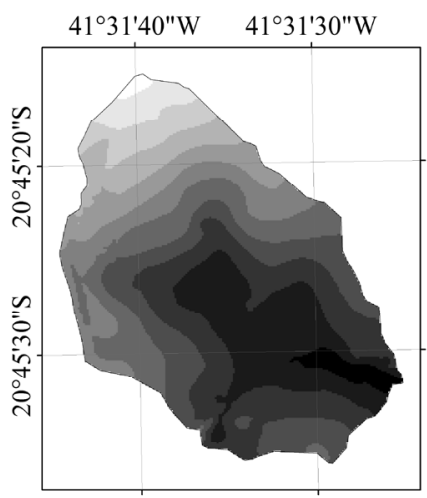

(a)

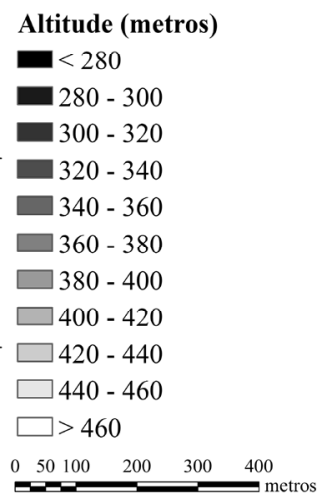

$0 \quad 50100 \quad 200 \quad 300 \quad 400$

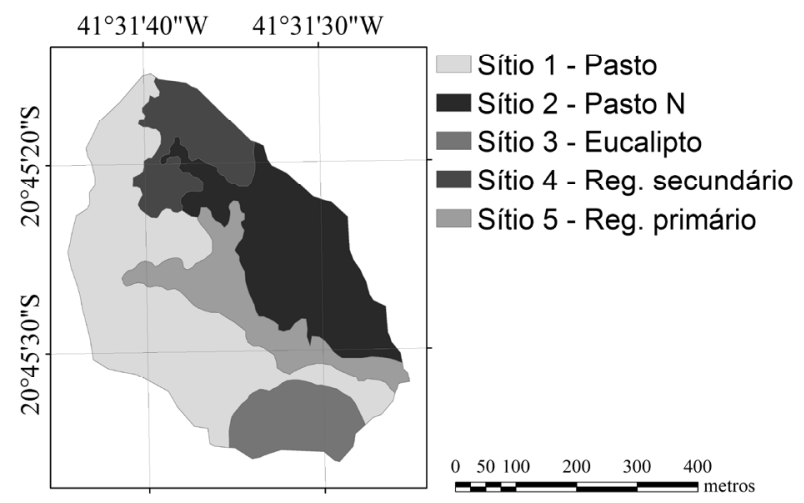

(b)

Fonte: Elaboração dos autores.

O solo da microbacia foi classificado como Latossolo Vermelho-Amarelo (LVa). Com relação ao uso e ocupação do solo, constataram-se cinco diferentes tipos de cobertura, que também foram mapeados por meio de GPS geodésico estático (modelo GTR-A da TechGeo®) (Figura 1b).

Em cada área com deferente cobertura do solo, foram realizadas amostragens para determinação das características físicas do solo (três repetições) até 60 cm de profundidade. Através da EMBRAPA (1997) foram determinados os seguintes atributos físicos do solo (Tabela 1): granulometria; densidade do solo, densidade de partículas, macro e microporosidade. A taxa de infiltração de água no solo $\left(\mathrm{T}_{\mathrm{ie}}\right)$ foi obtida em experimentos de campo, utilizando o método de anéis concêntricos (BRANDÃO et al., 2006). Além disso foram determinadas as curvas de retenção de água no solo nas tensões de 6, 10, 30, 100, 800 e $1.500 \mathrm{kPa}$ (Tabela 2). 
Tabela 1. Atributos físicos do solo nos sítios da microbacia hidrográfica do córrego Jaqueira, ES.

\begin{tabular}{lccccc}
\hline \multicolumn{1}{c}{ Atributo } & \multicolumn{5}{c}{ Sítio } \\
\cline { 2 - 6 } \multicolumn{1}{c}{$\mathbf{1}$} & $\mathbf{2}$ & $\mathbf{3}$ & $\mathbf{4}$ & $\mathbf{5}$ \\
\hline Teor de areia $\left(\mathrm{g} \mathrm{kg}^{-1}\right)$ & 490,75 & 482,16 & 467,48 & 465,49 & 618,34 \\
Teor de silte $\left(\mathrm{g} \mathrm{kg}^{-1}\right)$ & 96,48 & 72,25 & 102,11 & 135,27 & 91,78 \\
Teor de argila $\left(\mathrm{g} \mathrm{kg}^{-1}\right)$ & 412,77 & 445,60 & 430,41 & 399,24 & 289,88 \\
Classificação textural & Argilosa & Argilosa & Argilosa & Argilosa & Média \\
Densidade do solo $\left(\mathrm{kg} \mathrm{dm}^{-3}\right)$ & 1,39 & 1,36 & 1,35 & 1,35 & 1,48 \\
Densidade de partículas $\left(\mathrm{kg} \mathrm{dm}^{-3}\right)$ & 2,60 & 3,05 & 2,68 & 2,91 & 2,47 \\
Microporosidade $\left(\mathrm{m}^{3} \mathrm{~m}^{-3}\right)$ & 0,31 & 0,32 & 0,33 & 0,34 & 0,27 \\
Macroporosidade $\left(\mathrm{m}^{3} \mathrm{~m}^{-3}\right)$ & 0,15 & 0,24 & 0,18 & 0,19 & 0,14 \\
Taxa de infiltração estável $\left(\mathrm{mm} \mathrm{h}^{-1}\right)$ & 50,1 & 163,4 & 184,1 & 119,0 & 439,1 \\
\hline
\end{tabular}

Fonte: Elaboração dos autores.

Tabela 2. Curva de retenção nos cinco sítios da microbacia hidrográfica do córrego Jaqueira, ES.

\begin{tabular}{cccccc}
\hline \multirow{2}{*}{ Tensão (kPa) } & \multicolumn{5}{c}{ Umidade $\left(\mathbf{c m}^{\mathbf{3}} \mathbf{c m}^{-3}\right)$} \\
\cline { 2 - 5 } & Sítio 1 & Sítio 2 & Sítio 3 & Sítio 4 & Sítio 5 \\
\hline 6 & 0,3062 & 0,3195 & 0,3251 & 0,3410 & 0,2728 \\
10 & 0,2809 & 0,2911 & 0,3031 & 0,3054 & 0,2598 \\
30 & 0,2433 & 0,2534 & 0,2588 & 0,2611 & 0,2136 \\
100 & 0,2391 & 0,2454 & 0,2376 & 0,2538 & 0,2116 \\
800 & 0,2061 & 0,2225 & 0,2156 & 0,2253 & 0,1645 \\
1500 & 0,1928 & 0,2057 & 0,2034 & 0,2040 & 0,1520 \\
\hline
\end{tabular}

Fonte: Elaboração dos autores.

\section{Monitoramento hidrológico}

O monitoramento da precipitação e vazão das águas na foz da microbacia foi realizado entre março de 2009 e janeiro de 2010, em intervalos de 3 minutos, por meio de estação pluviográfica automática e, no exutório, um vertedor triangular aliado em um linígrafo de bóia. Os registros forneceram os hidrogramas de escoamento do curso d'água durante cada evento de precipitação. Alâmina de escoamento superficial e a vazão máxima foram calculadas a partir da separação entre o hidrograma de escoamento superficial e o de fluxo subterrâneo, utilizando o método de Barnes (ZANETTI et al., 2009b). Na Tabela 3 são apresentados os oito maiores eventos observados de precipitação utilizados no HidroBacia, com suas respectivas vazões máximas e lâminas de escoamento superficial geradas. 
Tabela 3. Eventos de precipitação selecionados para avaliação com os respectivos escoamentos superficiais na microbacia hidrográfica do córrego Jaqueira, ES.

\begin{tabular}{cccccccc}
\hline Evento & Data & $\begin{array}{c}\text { Duração } \\
(\mathbf{m i n})\end{array}$ & $\begin{array}{c}\text { Lâmina } \\
(\mathbf{m m})\end{array}$ & $\begin{array}{c}\mathbf{I p}_{\text {máx }} \\
\left(\mathbf{m m ~ h}^{-1}\right)\end{array}$ & $\begin{array}{c}\mathbf{I p} \\
\left(\mathbf{m m} \mathbf{h}^{-1}\right)\end{array}$ & $\begin{array}{c}\mathbf{L E S} \\
(\mathbf{m m})\end{array}$ & $\begin{array}{c}\mathbf{Q}_{\text {máx }} \\
\left(\mathbf{L ~ s}^{-1}\right)\end{array}$ \\
\hline 1 & $19 / 07 / 2009$ & 96 & 21,1 & 106,7 & 13,2 & 0,03 & 4,42 \\
2 & $19 / 10 / 2009$ & 57 & 29,8 & 91,4 & 31,4 & 0,03 & 4,33 \\
3 & $27 / 10 / 2009$ & 273 & 37,8 & 45,7 & 8,3 & 0,12 & 4,02 \\
4 & $29 / 11 / 2009$ & 36 & 15,2 & 40,6 & 25,4 & 0,05 & 4,30 \\
5 & $14 / 12 / 2009$ & 114 & 11,9 & 30,5 & 6,3 & 0,02 & 1,98 \\
6 & $14 / 12 / 2009$ & 180 & 19,3 & 40,6 & 6,4 & 0,04 & 4,08 \\
7 & $18 / 12 / 2009$ & 168 & 19,8 & 45,7 & 7,0 & 0,04 & 3,35 \\
8 & $21 / 12 / 2009$ & 69 & 13,2 & 71,1 & 11,5 & 0,02 & 3,18 \\
\hline
\end{tabular}

I $p_{\text {máx }}$ - intensidade de precipitação máxima; Ip - intensidade de precipitação média; LES - lâmina de escoamento superficial, $Q_{\text {máx }}$ - vazão máxima de escoamento superficial.

Fonte: Elaboração dos autores.

\section{Aplicação do método racional}

A vazão máxima estimada pelo Método Racional foi calculada pela Equação 1.

$$
\mathrm{Q}_{\max }=\frac{\mathrm{Ci}_{\mathrm{m}} \mathrm{A}_{\mathrm{d}}}{360}
$$

Em que,

$\mathrm{Q}_{\text {máx }}$ - vazão máxima de escoamento superficial, $\mathrm{m}^{3}$ $\mathrm{S}^{-1}$;

C - coeficiente de escoamento superficial, adimensional;

$\mathrm{i}_{\mathrm{m}}$ - intensidade máxima de precipitação, $\mathrm{mm} \mathrm{h}^{-1}$;

$A_{d}$ - área de drenagem da bacia hidrográfica, ha.

O coeficiente de escoamento superficial (C) foi estimado com base no Soil Conservation Service - USDA, obtendo-se valor igual a 0,59. Para a determinação da intensidade máxima de precipitação $\left(i_{m}\right)$, foram considerados os valores mensurados pela equação 2 .

$$
\mathrm{i}_{\mathrm{m}}=\frac{\mathrm{PT}}{\mathrm{t}}
$$

Em que,

$\mathrm{i}_{\mathrm{m}}$ - intensidade máxima de precipitação, $\mathrm{mm} \mathrm{h}^{-1}$;

$\mathrm{T}$ - precipitação do evento, mm;

$\mathrm{t}$ - duração da precipitação, min.

\section{Aplicação do método do número da curva}

Para o cálculo da lâmina de escoamento superficial, foi utilizada a Equação 3.

$$
\mathrm{LES}=\frac{\left(\mathrm{PT}-0,2 \mathrm{~S}_{\mathrm{p}}\right)^{2}}{\left(\mathrm{PT}+0,8 \mathrm{~S}_{\mathrm{p}}\right)}
$$

Em que,

LES - lâmina de escoamento superficial, mm;

$\mathrm{S}_{\mathrm{p}}$ - infiltração potencial, $\mathrm{mm}$.

Para se determinar a infiltração potencial $\left(\mathrm{S}_{\mathrm{p}}\right)$ foi utilizadaa Equação 4.

$$
\mathrm{S}_{\mathrm{p}}=\frac{25400}{\mathrm{CN}}-254
$$

Onde CN é o número da curva, adimensional, cujo valor pode variar de 0 a 100 , em função do uso e manejo das culturas, grupo do solo, condição hidrológica e umidade antecedente do solo.

$\mathrm{O}$ valor correspondente a $\mathrm{CN}$ de cada uso e ocupação da terra foi obtido de acordo com as tabelas propostas por Mockus (1972). Os critérios de estabelecidos por Pruski et al. (1997a) para classificação do solo e enquadramento nas classes, "B"e "C", também foram considerados. Além disso, quando houve necessidade, cada evento de 
precipitação analisado teve seu $\mathrm{CN}$ corrigido de acordo com a condição de umidade antecedente.

\section{Aplicação do hidrobacia}

Para a utilização do modelo HidroBacia são necessárias imagens temáticas do MDE, ao sentido do escoamento e à rede de drenagem numérica da bacia, as quais foram discretizadas em células/ pixels uniformes, quadradas (formato raster) e com dimensões de $3 \times 3$ metros, utilizado o software
ArcView 3.2a. Além disto, foram processadas imagens das características físicas do solo e, também, da cobertura vegetal (ICV), capacidade de armazenamento superficial (ARM) e coeficiente de rugosidade do terreno (n), de cada área da microbacia com diferente uso do solo (Tabela 4), considerando-se, portanto, a variabilidade espacial dos parâmetros. Estes foram estimados utilizando o próprio banco de dados do HidroBacia, a partir de informações de Woolhiser, Smith e Goodrich (1990), Morgan, Quinton e Rickison (1998), Beasley e Huggins (1981) e Onstad (1984).

Tabela 4. Valores de interceptação pela cobertura vegetal (ICV), capacidade de armazenamento superficial (ARM) e do coeficiente de rugosidade do terreno (n), para os sítios da microbacia hidrográfica do córrego Jaqueira, ES.

\begin{tabular}{cccccc}
\hline \multirow{2}{*}{ Variáveis } & \multicolumn{5}{c}{ Sítio } \\
\cline { 2 - 6 } & $\mathbf{1}$ & $\mathbf{2}$ & $\mathbf{3}$ & $\mathbf{4}$ & $\mathbf{5}$ \\
\hline $\mathrm{ICV}(\mathrm{mm})$ & 2,0 & 2,0 & 1,0 & 2,5 & 2,5 \\
$\mathrm{ARM}(\mathrm{mm})$ & 1,6 & 1,7 & 0,9 & 0,8 & 1,0 \\
$\mathrm{n}\left(\mathrm{s} \mathrm{m}^{-1 / 3}\right)$ & 0,10 & 0,10 & 0,20 & 0,15 & 0,15 \\
\hline
\end{tabular}

Fonte: Elaboração dos autores.

O HidroBacia utiliza a equação de GreenAmpt-Mein-Larson (GAML) para determinação da taxa de infiltração de água no solo. Esta equação é fundamentada em processos físicos, entretanto, necessita-se de parâmetros cuja determinação envolve métodos empíricos. Assim, buscando melhor ajuste dos parâmetros na equação, realizouse a adequação dos parâmetros de entrada conforme sugerido por Brandão et al. (2006), resultando na equação:

$$
\mathrm{T}_{\mathrm{i}}=\mathrm{K}_{\mathrm{w}}\left(1+\Psi_{\mathrm{f}} \frac{\left(\theta_{\mathrm{w}}-\theta_{\mathrm{i}}\right)}{\mathrm{I}}\right)
$$

em que,

$\mathrm{T}_{\mathrm{i}}$ - taxa de infiltração de água no solo, $\mathrm{mm} \mathrm{h}^{-1}$;

$\mathrm{K}_{\mathrm{w}}$ - condutividade hidráulica do solo saturado, $\mathrm{mm}$ $\mathrm{h}^{-1}$;

$\Psi_{\mathrm{f}}$ - potencial matricial do solo na frente de umedecimento, mm;
I - infiltração acumulada, mm;

$\theta_{\mathrm{w}}$ - umidade do solo na zona de transmissão, $\mathrm{cm}^{3}$ $\mathrm{cm}^{-3}$;

$\theta_{\mathrm{i}}$ - umidade do solo no início do processo de infiltração, $\mathrm{cm}^{3} \mathrm{~cm}^{-3}$.

Nesse sentido, para a realização das simulações foram utilizados diferentes métodos de obtenção dos parâmetros: $\Psi_{\mathrm{f}}, \mathrm{K}_{\mathrm{w}}$ e $\theta_{\mathrm{w}}$.

$\mathrm{Na}$ obtenção de $\Psi_{\mathrm{f}}$ foram considerados três métodos: RB (RAWLS; BRAKENSIEK, 1983), ML (MEIN; LARSON, 1973), e CE (CECÍlIO et al., 2007), descritos pelas Equações 6, 7 e 8, respectivamente.

$$
\Psi_{\mathrm{f}}=0,01 \mathrm{e}^{a}
$$

Sendo, 
$\alpha=6,531-7,33 \theta_{\mathrm{w}}+15,8 \mathrm{Ag}^{2}+3,81 \theta_{\mathrm{w}}{ }^{2}+3,4 \mathrm{Ar} \mathrm{Ag}-4,98 \mathrm{Ar} \theta_{\mathrm{w}}+$ $\alpha=6,531-7,33 \theta_{\mathrm{w}}+15,8 \mathrm{Ag}^{2}+3,81 \theta_{\mathrm{w}}{ }^{2}+3,4 \mathrm{Ar} \mathrm{Ag}-4,98 \mathrm{Ar} \theta_{\mathrm{w}}+$ $+16,1 \mathrm{Ar}^{2} \theta_{\mathrm{w}}{ }^{2}+16 \mathrm{Ag}^{2} \theta_{\mathrm{w}}{ }^{2}-14 \mathrm{Ar}^{2} \mathrm{Ag}-34,8 \mathrm{Ag}^{2} \theta_{\mathrm{w}}-8 \mathrm{Ar}^{2} \theta_{\mathrm{w}}$

Em que,

$\mathrm{Ag}$ - teor de argila no solo, $\mathrm{kg} \mathrm{kg}^{-1}$;

$\mathrm{Ar}$ - teor de areia no solo, $\mathrm{kg} \mathrm{kg}^{-1}$;

$$
\Psi_{\mathrm{f}}=\int_{0,01}^{1,0} \Psi \mathrm{dK}_{\mathrm{r}}
$$

Em que,

$\Psi$ - potencial matricial, $\mathrm{mm}$;

$\mathrm{K}_{\mathrm{r}}$ - condutividade hidráulica relativa, adimensional.

$\Psi_{\mathrm{f}}=\frac{\Psi_{\mathrm{b}}(2+3 \lambda)\left[\mathrm{K}_{\mathrm{r}}\left(\theta_{\mathrm{w}}\right)^{\frac{3 \lambda+1}{3 \lambda+2}}-\mathrm{K}_{\mathrm{r}}\left(\theta_{\mathrm{i}}\right)^{\frac{3 \lambda+1}{3 \lambda+2}}\right]}{\left[\mathrm{K}_{\mathrm{r}}\left(\theta_{\mathrm{w}}\right)-\mathrm{K}_{\mathrm{r}}\left(\theta_{\mathrm{i}}\right)\right](3 \lambda+1)}$

Em que,

$\Psi_{\mathrm{b}}$ - pressão de borbulhamento, mm;

$\lambda$ - índice de distribuição do tamanho de poros, adimensional.

Para a estimação de $\mathrm{K}_{\mathrm{w}}$ utilizaram-se seis diferentes valores: $\mathrm{T}_{\mathrm{ie}}(\mathrm{K} 1), \mathrm{T}_{\mathrm{ie}} / 2(\mathrm{~K} 2), \mathrm{T}_{\mathrm{ie}} / 3(\mathrm{~K} 3)$, $\mathrm{T}_{\mathrm{ie}} / 4(\mathrm{~K} 4), \mathrm{T}_{\mathrm{ie}} / 5(\mathrm{~K} 5)$ e $\mathrm{T}_{\mathrm{ie}} / 6(\mathrm{~K} 6)$. Para $\theta_{\mathrm{w}}$ foram considerados quatro diferentes valores: $\theta_{\mathrm{s}}\left(\mathrm{q}_{100}\right)$; $0,90 \theta_{\mathrm{s}}\left(\mathrm{q}_{90}\right) ; 0,85 \theta_{\mathrm{s}}\left(\mathrm{q}_{85}\right)$, e $0,80 \theta_{\mathrm{s}}\left(\mathrm{q}_{80}\right)$.

Com base nas opções de dados de entrada para o modelo de GAML apresentadas anteriormente, foram obtidas 72 combinações distintas de parâmetros de entrada para a realização das simulações no software HidroBacia. Assim, utilizando os eventos de precipitação apresentados na Tabela 2, realizaram-se 576 simulações com o HidroBacia.

\section{Análise estatística}

A avaliação consistiu em comparar os dados observados de lâmina de escoamento superficial e vazão máxima na foz da microbacia com as simulações realizadas pelo HidroBacia, MR e MNC. Para tanto foram utilizados dois índices estatísticos: o erro absoluto médio (EAM) e a raiz do erro quadrado médio (REQM).

$$
\begin{gathered}
\text { EAM }=\frac{\sum_{i=1}^{n}\left|O_{i}-E_{i}\right|}{n} \\
\text { REQM }=\sqrt{\frac{\sum_{i=1}^{n}\left(O_{i}-E_{i}\right)^{2}}{n}}
\end{gathered}
$$

Em que,

$\mathrm{O}_{\mathrm{i}}$ - valor observado experimentalmente;

$\mathrm{E}_{\mathrm{i}}$ - valor estimado pelos modelos;

n - número de observações;

\section{Resultados e Discussão}

Com relação às simulações obtidas com o HidroBacia, como o número de combinações testadas foi elevado (72 combinações), são apresentados na Tabela 5 os índices estatísticos relativos àquelas que promoveram as melhores estimativas da vazão máxima $\left(\mathrm{Q}_{\text {máx }}\right)$ e da lâmina de escoamento superficial (LES). De modo geral, os índices estatísticos apresentaram alta variação. Contudo, percebeu-se que o HidroBacia é mais preciso na estimação da LES, já que os coeficientes calculados se apresentaram melhores do que quando se estimou a $\mathrm{Q}_{\text {máx }}$. Isso se deve ao fato de LES ser obtida a partir da divisão do volume de escoamento superficial pela área da bacia (área de drenagem), enquanto a $\mathrm{Q}_{\text {máx }}$ é um valor pontual do hidrograma, sendo mais difícil de ser estimado. Além disso, para o cálculo da $\mathrm{Q}_{\text {máx }}$ é utilizado o método de ondas cinemáticas (CHOW; MAIDMENT; MAYS, 1998), que tenta simular a propagação do hidrograma considerando a declividade e a rugosidade do terreno; de forma que se estas informações forem mal estimadas, o resultado da $Q_{\text {máx }}$ fica prejudicado, mas a LES não. 
Tabela 5. Indicadores do ajuste entre dados simulados pelo HidroBacia e observados de lâmina de escoamento superficial (LES) e vazão máxima $\left(Q_{\text {máx }}\right)$, para 8 eventos de precipitação observados na microbacia hidrográfica do córrego Jaqueira, ES.

\begin{tabular}{ccccc}
\hline \multirow{2}{*}{ Combinação } & \multicolumn{2}{c}{ LES } & \multicolumn{2}{c}{ Q $_{\text {máx }}$} \\
\cline { 2 - 5 } & EAM (mm) & REQM (mm) & EAM (L s $\left.\mathbf{~}^{-1}\right)$ & REQM (L s $\left.\mathbf{~}^{-1}\right)$ \\
\hline ML-K3- $\theta_{90}$ & 0,16 & 0,21 & 10,50 & 13,95 \\
ML-K3- $\theta_{85}$ & 0,35 & 0,37 & 25,68 & 27,29 \\
ML-K4- $\theta_{100}$ & 0,36 & 0,38 & 26,52 & 28,26 \\
CE-K4- $\theta_{100}$ & 0,32 & 0,32 & 22,00 & 22,92 \\
CE-K4- $\theta_{90}$ & 0,20 & 0,23 & 11,86 & 14,75 \\
CE-K4- $\theta_{85}$ & 0,14 & 0,17 & 7,14 & 10,04 \\
CE-K4- $\theta_{80}$ & 0,09 & 0,12 & 5,91 & 6,46 \\
\hline
\end{tabular}

Fonte: Elaboração dos autores.

As combinações entre parâmetros da equação de GAML que melhor representaram a LES e $\mathrm{Q}_{\text {máx }}$, apresentadas na Tabela 5 foram: ML-K3- $\mathrm{q}_{90}$; ML-

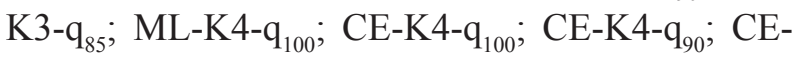
$\mathrm{K} 4-\mathrm{q}_{85}$ e CE-K4- $\mathrm{q}_{80}$. Para o presente trabalho, as equações que apresentaram melhor desempenho para se estimar o potencial matricial na frente de umedecimento foram ML e, principalmente, CE. A Equação ML apresentou melhor desempenho quando associada com $\mathrm{K}_{\mathrm{w}}$, correspondente a um terço ou um quarto da $\mathrm{T}_{\text {ie }}$ obtida pelo método do infiltrômetro de anéis e $\theta_{\mathrm{w}}$ equivalente a $\theta_{\mathrm{s}}, 0,90$ $\theta_{\mathrm{s}}$ ou $0,85 \theta_{\mathrm{s}}$, enquanto a Equação $\mathrm{CE}$ somente apresentou melhor desempenho quando associada a $\mathrm{K}_{\mathrm{w}}$, correspondente a um quarto da $\mathrm{T}_{\mathrm{ie}}$ e todas as frações de $\theta_{w}$, ou seja, equivalente a $\theta_{s}, 0,90 \theta_{s}$, $0,85 \theta_{\mathrm{s}}$ ou $0,80 \theta_{\mathrm{s}}$. As combinações que utilizaram a RB para estimar o potencial matricial simularam escoamento superficial extremamente alto. Isso se deve, corroborando Bezerra e Rodrigues (2006), aos baixos valores de potencial matricial calculados pela equação, fato que contribui para menor estimava da infiltração de água no solo.

Zanetti et al. (2009b) avaliaram combinações dos parâmetros de entrada do modelo de GAML por meio de simulações utilizando o HidroBacia. Dentre as seis combinações mais favoráveis, tanto para estimação da LES quanto da $\mathrm{Q}_{\text {máx }}$, $\mathrm{O}$ potencial matricial na frente de umedecimento
$\left(\Psi_{\mathrm{f}}\right)$ foi estimado por RB e CE. A Equação RB somente apresentou melhor desempenho quando associada com $\mathrm{K} 1$ ou $\mathrm{K} 2$, e $\theta_{85}$ ou $\theta_{80}$, enquanto a CE apresentou melhor desempenho somente quando associada com K1 e $\theta_{85}$ ou $\theta_{80}$. A equação $\mathrm{CE}$ se destacou tanto no presente trabalho quanto nas avaliações de Zanetti et al. (2009b); enquanto as combinações que utilizaram RB não apresentaram a mesma eficiência observada pelos autores. Esse fato justifica-se pela equação RB ser empírica, derivada de dados experimentais, acabando por não ser aplicável em qualquer solo (CECÍLIO et al., 2007; ZONTA et al., 2010), como o do presente estudo.

Pode-se observar uma tendência quanto aos valores ótimos de $\mathrm{K}_{\mathrm{w}}$, pois ao se estimar o $\Psi_{\mathrm{f}}$ pelas Equações ML e CE, as combinações que melhor estimaram o escoamento superficial foram aquelas que utilizaram um terço e um quarto da $\mathrm{T}_{\text {ie }}(\mathrm{K} 3 \mathrm{e}$ K4). Esses valores evidenciam que a infiltração obtida pelo infiltrômetro de anéis é maior que aquela que ocorre naturalmente, fazendo com que a $\mathrm{T}_{\mathrm{ie}}$ seja superestimada, corroborando com alguns trabalhos que os avaliaram para determinação da $\mathrm{T}_{\mathrm{ie}}$, como Pruski et al. (1997b), Simões, Figueirêdo e Silva (2005) e Brandão et al. (2006).

Os valores de $\mathrm{Q}_{\text {máx }}$ estimados pelo $\mathrm{MR}$ e os valores de LES estimados pelo MNC, juntamente com os resultados dos índices estatísticos 
calculados, são apresentados na Tabela 6. Nota-se que tanto os resultados obtidos pelo MR quanto pelo MNC superestimam os valores observados, de forma semelhante ao estudo realizado por Silva et al. (2009a) na microbacia do Palmital, em ViçosaMG.

Tabela 6. Vazão máxima estimada pelo $M R\left(Q_{\text {maxx }} M R\right)$ e lâmina de escoamento superficial estimada pelo $M N C$ (LES MNC) para 8 eventos de precipitação observados na microbacia hidrográfica do córrego Jaqueira, ES.

\begin{tabular}{cccc}
\hline Evento & Data & $\mathbf{Q}_{\text {máx }} \mathbf{M R}\left(\mathbf{L} \mathbf{~ s}^{-1}\right)$ & LES MNC (mm) \\
\hline 1 & $19 / 07 / 2009$ & 574,72 & 10,37 \\
2 & $19 / 10 / 2009$ & 776,69 & 10,45 \\
3 & $27 / 10 / 2009$ & 308,27 & 6,13 \\
4 & $29 / 11 / 2009$ & 830,90 & 12,46 \\
5 & $14 / 12 / 2009$ & 384,45 & 13,79 \\
6 & $14 / 12 / 2009$ & 526,18 & 10,97 \\
7 & $18 / 12 / 2009$ & 390,11 & 2,11 \\
8 & $21 / 12 / 2009$ & 520,15 & 13,27 \\
\hline \multirow{2}{*}{ Índices estatísticos } & EAM & 535,23 & 9,90 \\
& REQM & 562,73 & 10,57 \\
\hline
\end{tabular}

Fonte: Elaboração dos autores.

Os resultados corroboram com afirmações de Bonta e Rao (1992), Silva et al. (2006a), ConesaGarcia et al. (2010), Haddad et al. (2010) e Jabur e Rizzi (2011) de que o MR é uma metodologia que superestima a vazão máxima de escoamento superficial. Uma das justificativas para os resultados obtidos pelo MR se refere ao coeficiente de escoamento superficial $(\mathrm{C}=0,59)$, utilizado no cálculo da $\mathrm{Q}_{\text {máx }}$, uma vez que a vazão estimada é diretamente proporcional a esse valor. Entretanto, o coeficiente $\mathrm{C}$ é empírico possuindo tabelas específicas, podendo não ser representativo das condições experimentais. Parak e Pegram (2006), assim como Young, Mcenroe e Rome (2009) criticam a falta de pesquisas relacionadas à determinação do fator $\mathrm{C}$ e sugerem a condução de estudos para sua determinação experimental antes da utilização do MR, em bacias hidrográficas. Salienta-se também que o MR apresenta algumas pressuposições que não são observadas na realidade e, por não considerar alguns parâmetros nos processos de escoamento, contribui para superestimar as vazões máximas. Além de considerar que toda a área da bacia hidrográfica contribui simultaneamente, com o escoamento superficial na seção de deságue (provocado por uma precipitação com intensidade constante e uniforme em toda a bacia), desconsidera a influência de muitos componentes do ciclo hidrológico no processo de formação do escoamento superficial, como a interceptação pela cobertura vegetal, o armazenamento superficial, a infiltração de água no solo, o perfil de precipitação, a rede drenagem, a rugosidade do terreno e a umidade inicial do solo (SILVA et al., 2009a).

A diferença entre os valores de LES observados e estimados pelo MNC (Tabela 6) pode ser consequência do processo de desenvolvimento deste método, já que foram utilizados dados regionais do meio-oeste dos Estados Unidos para sua elaboração (PONCE; HAWKINS, 1996). O MNC não leva em consideração a variabilidade espacial e temporal da infiltração de água no solo, mas descreve tendências médias, o que impede que ele seja previsível. Além disso, é descrito por Ponce e Hawkins (1996) que o 
MNC possui uma capacidade de resposta a apenas quatro parâmetros em uma bacia hidrográfica: tipo, uso, manejo e condição hidrológica. Para o enquadramento dos parâmetros em suas respectivas classes, existe a possibilidade de falhas. Este fato pode ser notado no enquadramento do tipo de solo, pela $\mathrm{T}_{\text {ie }}$ já que a amplitude das classes é alta (SILVA et al., 2006a). Segundo Boughton (1989), a seleção dos valores do número da curva é um dos principais erros para estimativa do escoamento, sendo a resposta a extremamente sensível, pois alterações de 15 a $20 \%$ nos valores adotados, representa em uma estimativa falsa da lâmina de escoamento superficial, ou seja, compromete os resultados. Por fim, quando comparados com outros resultados obtidos pelo $\mathrm{MNC}$, superestimaram a lâmina de escoamento superficial (SARTORI; GENOVEZ; LOMBARDI NETO, 2005; SILVA et al., 2006a; 2009a; CARLESSO et al., 2011; DALIRI et al., 2011).

Usualmente, muito dos parâmetros de entrada dos modelos MR e MNC, e até mesmo alguns usados pelo HidroBacia, não foram definidos para regiões climáticas brasileiras e, caso tenham sido, apresentam pouca representatividade para toda sua extensão territorial. Isso se confirma ao utilizar os dados de coeficiente de escoamento superficial (C) recomendado pelo Soil Conservation Service - USDA, para enquadramento das condições da vegetação; realizar a classificação hidrológica do solo, por meio das recomendações do Soil Conservation Service - USDA, para escolher o tipo de solo e determinar o valor do número da curva; e adotar parâmetros ajustados para as condições de clima temperado, como: intercepção pela cobertura vegetal, capacidade de armazenamento superficial, coeficiente de rugosidade do terreno, entre outros.

Salienta-se, ainda que, a classificação hidrológica dos solos brasileiros, está sendo realizada, uma vez que existem classes com diferentes potenciais de escoamento superficial, os quais não pertencem aos grupos hidrológicos apresentados nas tabelas do Soil Conservation Service (SARTORI; GENOVEZ;
LOMBARDI NETO, 2005). No entanto, esta nova classificação deve ser utilizada com cautela, já que as informações obtidas para seu desenvolvimento foram baseadas nos solos do Estado de São Paulo e, com isso, não representam diversas regiões do país, o que indica a necessidade de se realizar levantamentos para toda a extensão territorial brasileira.

Em geral, existe uma tendência em utilizar modelos hidrológicos simples e com poucos parâmetros de entrada, como o MR e o MNC, já que sua codificação é fácil de ser executada e o esforço de coleta de dados é menor (LIMBRUNNER; VOGEL; CHAPRA, 2005). Ainda segundo Limbrunner; Vogel e Chapra (2005), estes modelos são úteis para o planejamento e gestão, podendo ser utilizados em tomadas de decisão sem que haja grandes despesas na coleta de dados e conhecimentos técnicos necessários para a utilização de modelos mais complexos. Em contrapartida, modelos hidrológicos simples não são adequados para alguns fins científicos, visto que, ao tentar representar diversos processos hidrológicos, o modelo deveria considerar os mais variados fatores, como tipo de solo, clima, cobertura vegetal, umidade antecedente do solo, processo de infiltração de água no solo, interceptação pela cobertura vegetal, entre outros. Além disto, ao se estudar o movimento das águas no solo, deve-se aumentar o nível de detalhamento da investigação, visto que outros fatores, como atributos físicos e químicos, exercem influência direta sobre o escoamento de água no solo, seja em grande ou pequena escala, no tempo e espaço.

Portanto, mesmo com a pouca eficiência apresentada nas simulações da $\mathrm{Q}_{\text {máx }}$ e da LES (quando comparado aos dados observados - Tabela 6), pode-se utilizar o MR e MNC para realizar previsões futuras de forma rápida e com baixo investimento, o que possibilitaria uma tomada de decisão praticamente em tempo instantâneo, porém, com baixa precisão. Em contrapartida, os resultados dos índices estatístico entre os dados observados e simulados pelo HidroBacia (Tabela 5) revelam 
que o modelo se comporta de forma mais precisa e, ainda, apresenta a vantagem de considerar a variação espacial dentro de uma bacia hidrográfica (Figura 2). Dessa forma, o HidroBacia apresentase como um modelo que pode e deve ser utilizado em estudos hidrológicos; no entanto, necessita de diversos parâmetros de entrada, o que pode limitar seu uso devido às dificuldades de obtenção dos mesmos.

Contudo, ressalta-se a necessidade de se realizar estudos básicos para adequação dos parâmetros de entrada, tanto para os modelos importados quanto para os nacionais. Ressalta-se, ainda, a necessidade de se testar mais o modelo HidroBacia para confirmar a sua eficácia na estimação da $\mathrm{Q}_{\text {máx }}$ e da LES.

\section{Conclusões}

1. Os resultados obtidos pelo MR e MNC superestimam a $\mathrm{Q}_{\text {máx }}$ e a LES;

2. Recomenda-se o uso do HidroBacia para estimação da $\mathrm{Q}_{\text {máx }}$ e da LES;

3. Dentre as combinações de dados de entrada da Equação de GAML avaliadas, as que melhor se comportaram foram: Equação de Mein e Larson (1973) associada a $K_{w}$ correspondente a $T_{i e} / 3$ e $\theta_{\mathrm{w}}$ equivalente a $0,90 \theta_{\mathrm{s}}$ ou $0,85 \theta_{\mathrm{s}}$, ou associada a $\mathrm{K}_{\mathrm{w}}$ correspondente a $\mathrm{T}_{\mathrm{ie}} / 4$ e $\theta_{\mathrm{w}}$ equivalente a $\theta_{\mathrm{s}}$; e a Equação de Cecílio et al. (2007) associada a $\mathrm{K}_{\mathrm{w}}$ correspondente a $\mathrm{T}_{\mathrm{ie}} / 4$ e todas as opções de $\theta_{\mathrm{w}}$ testadas $\left(\theta_{\mathrm{s}}, 0,90 \theta_{\mathrm{s}}, 0,85 \theta_{\mathrm{s}}\right.$ ou $\left.0,80 \theta_{\mathrm{s}}\right)$.

\section{Agradecimentos}

Ao Conselho Nacional de Desenvolvimento Científico e Tecnológico - CNPq (processo $n^{\circ}$ 480975/2007-8) pelo apoio financeiro e à da Fundação de Amparo à Pesquisa do Espírito Santo - FAPES, pelo apoio financeiro (processo $\mathrm{n}^{\circ}$ 35613017/06) e pela concessão da bolsa de estudos em nível de mestrado (Processo no 41894065 , edital 003/2008 PROCAP I).

\section{Referências}

BEASley, D. B.; HUGGINS, L. F. Answers: user's manual. Chicago: United States Environmental protection Agency, Region V, 1981. 51 p.

BERTOL， I.; BARBOSA，F. T.; FABIAN, E. L.; PEGORARO, R.; ZAVASCHI, E.; GONZÁLEZ, A. P.; VÁZQUEZ, E. V. Escoamento superficial em diferentes sistemas de manejo em um Nitossolo Háplico típico. Revista Brasileira de Engenharia Agrícola e Ambiental, Campina Grande, v. 12, n. 3, p. 243-250, 2008.

BERTOL, O. J.; RIZZI, N. E.; BERTOL, I.; ROLOFF, G. Perdas de solo e água e qualidade do escoamento superficial associadas à erosão entre sulcos em área cultivada sob semeadura direta e submetida às adubações mineral e orgânica. Revista Brasileira de Ciência do Solo, Viçosa, MG, v. 31, n. 4, p. 781-792, 2007.

BEZERRA, J. F. R.; RODRIGUES, S. C. Estudo do potencial matricial e geotêxteis aplicado à recuperação de um solo degradado, Uberlândia (MG). Caminhos de Geografia, Uberlânida, v. 6, n. 19, p. 160-174, 2006.

BONTA, J. V.; RAO, A. R. Estimating peak flows for small agricultural watersheds. Journal of Irrigation and Drainage Engineering, Reston, v. 118, n. 1, p. 122-137, 1992.

BOUGHTON, W. C. A review of the USDA SCS curve number method. Australian Journal of Soil Research, Victoria, v. 27, n. 3, p .511-523, 1989.

BRANDÃO, V. S.; CECÍLIO, R. A.; PRUSKI, F. F.; SILVA, D. D. Infiltração da água no solo. 3. ed. Viçosa, MG: UFV, 2006. 120 p.

CAO, H.; VERVOORT, R. W.; DABNEY, S. M. Variation in curve numbers derived from plot runoff data for New South Wales (Australia). Hydrological Processes, Malden, v. 25, n. 24, p. 3774-3789, 2011.

CARLESSO, R.; SPOHR, R. B.; ELTZ, F. L. F.; FLORES, C. H. Runoff estimation in southern Brazil based on Smith's modified model and the Curve Number method. Agricultural Water Management, Amsterdan, v. 98, n. 6, p. 1020-1026, 2011.

CECÍLIO, R. A.; MARTINEZ, M. A.; PRUSKI, F. F.; SILVA, D. D.; ATAÍDE, W. F. Substituição dos parâmetros do modelo de Green-Ampt-Mein-Larson para estimativa da infiltração em alguns solos do Brasil. Revista Brasileira de Ciência do Solo, Viçosa, v. 31, n. 5, p. 1141-1151, 2007.

CHOW, V. T.; MAIDMENT, D. R.; MAYS, L. W. Appied hydrology. New York: McGraw-Hill, 1988. 572 p. 
CONESA-GARCIA, C.; CASELLES-MIRALLES, V.; TOMAS, J. M. S.; GARCIA-LORENZO, R. Hydraulic geometry, gis and remote sensing, techniques against rainfall-runoff models for estimating flood magnitude in ephemeral fluvial systems. Remote Sensing, Basel, v. 2, n. 11, p. 2607-2628, 2010.

DALIRI, F.; SERAJI, H. S.; KHOLGHI, M.; AHMADI, H. Soil hydrologic group detection based on geomorphologic facieses and slope (Case study: Taleghan Watershed, Iran). Desert, Tehran, v. 16, n. 1, p. 69-75, 2011.

EMPRESA BRASILEIRA DE PESQUISA AGROPECUÁRIA - EMBRAPA. Manual de método e análise de solo. 2. ed. Rio de Janeiro: Ministério da Agricultura e do Abastecimento, 1997. 212 p.

HADDAD, K.; KUCZERA, G.; RAHMAN, A.; WEINMANN, P. E.; ZAMAN, M. Design flood estimation in ungauged catchments: a comparison between the probabilistic rational method and quantile regression technique for NSW. Australian Journal of Water Resources, Newcastle, v. 14, n. 2, p. 127-139, 2010.

JABUR, A.; RIZZI, N. Estimativas da vazão máxima e capacidade de saturação hídrica da bacia hidrográfica do alto do rio Ligeiro em Pato Branco, PR. Floresta, Curitiba, v. 41, n. 4, p. 833-846, 2011.

LIMBRUNNER, J. F.; VOGEL, R. M.; CHAPRA, S. C. Chapter 22: a parsimonious watershed model. In: SINGH, V. P.; FREVERT, D. F. Watershed models. Colorado: CRC Press, 2005. p. 549-567.

LOURENÇÃO, A.; HONDA, E. A. Influência do reflorestamento com essências nativas sobre a infiltração da água e a velocidade do escoamento superficial. Revista do Instituto Florestal e IF Série Registros, São Paulo, v. 1, n. 31, p. 33-37, 2007.

MEIN, R. G.; LARSON, C. L. Modeling infiltration during a steady rain. Water Resources Research, Washington, v. 9, n. 2, p. 384-394, 1973.

MOCKUS, V. Hydrology soil-cover complexes. In: SCS National Engineering Handbook, Section 4, Washington, Hidrology, Part 1, 1972.

MORGAN, R. P. C.; QUINTON, J. N.; RICKISON, R. J. EUROSEM: a user guide. Version 2. Silsoe: Silsoe College, 1998. 86 p.

OLIVEIRA, J. R.; PINTO, M. F.; SOUZA, W. J.; GUERRA, J. G. M.; CARVALHO, D. F. Erosão hídrica em um Argissolo Vermelho-Amarelo, sob diferentes padrões de chuva simulada. Revista Brasileira de Engenharia Agrícola e Ambiental, Campina Grande, v. 14, n. 2, p. 140-147, 2010.
ONSTAD, C. A. Depressional storage on tilled soil surfaces. Transactions of the American Society of Agricultural Engineers, St. Joseph, v. 27, n. 3, p. 729$732,1984$.

PARAK, M.; PEGRAM, G. G. S. The rational formula from the runhydrograph. Water $S A$, Gezina, v. 32, n. 2, p. 163-180, 2006.

PINTO, D. B. F.; SILVA, A. M.; MELLO, C. R.; COELHO, G. Qualidade da água do Ribeirão Lavrinha na região Alto Rio Grande - MG, Brasil. Ciência e Agrotecnologia, Lavras, v. 33, n. 4, p. 1145-1152, 2009.

PONCE, V. M.; HAWKINS, R. H. Runoff curve number: has it reached maturity? Journal of Hydrologic Engineering, West Lafayette, v. 1, n. 1, p. 11-19, 1996.

PRUSKI, F. F.; FERREIRA, P. A.; RAMOS, M. M.; CECON, P. R. A model to design level terraces. Journal of Irrigation and Drainage Engineering, New York, v. 123 , n. 1, p. 8-12, 1997a.

PRUSKI, F. F.; VENDRAME, V.; OLIVEIRA, E. F.; BALBINO, L. C.; FERREIRA, P. A.; WERLANG, L.; CARVALHO, L. T. Infiltração da água num Latossolo Roxo. Pesquisa Agropecuária Brasileira, Brasília, v. 32, n. 1, p. 77-84, 1997b.

RAWLS, W. J.; BRAKENSIEK, D. L. A procedure to predict Green and Ampt infiltration parameters. In: AMERICAN SOCIETY OF AGRICULTURAL ENGINEERS CONFERENCE ON ADVANCES IN INFILTRATION, 3., 1983, Saint Joseph, Proceedings... Saint Joseph: American Society of Agricultural Engineers, 1983. p. 102-112.

ROSATTO, H. G.; TOLON-BECERRA, A.; BOTTA, G.; PRESUTTI, M. E. Runoff estimation in small rural watersheds using DEMS in North West of Argentina. Soil and Tillage Research, Amsterdam, v. 112, n. 1, p. 8-17, 2011.

SAHU, R. K.; MISHRA, S. K.; ELDHO, T. I. An improved AMC-coupled runoff curve number model. Hydrological Processes, Malden, v. 24, n. 20, p. 28342839, 2010.

SARTORI, A.; GENOVEZ, A. M.; LOMBARDI NETO, F. Classificação hidrológica de solos brasileiros para a estimativa da chuva excedente com o método do serviço de conservação do solo dos Estados Unidos Parte 2: Aplicação. Revista Brasileira de Recursos Hídricos, Porto Alegre, v. 10, n. 4, p. 19-29, 2005.

SILVA, J. M. A.; PRUSKI, F. F.; RODRIGUES, L. N.; CECÍLIO, R. A. Modelo para a obtenção do hidrograma de escoamento superficial em bacias hidrográficas. 2 . Avaliação. Revista Brasileira de Ciências Agrárias, Recife, v. 4, n. 2, p. 192-197, 2009a. 
SILVA, J. M. A.; PRUSKI, F. F.; RODRIGUES, L. N.; CECÍLIO, R. A. Modelo para obtenção do hidrograma de escoamento superficial em bacias hidrográficas. 1 . Desenvolvimento e análise de sensibilidade. Revista Brasileira de Ciências Agrárias, Recife, v. 4, n. 1, p. 7884, 2009b.

SILVA, J. M. A.; PRUSKI, F. F.; SILVA, D. D.; CECÍLIO, R. A. Metodologia para obtenção do hidrograma de escoamento superficial em encostas e canais. Parte I: desenvolvimento e avaliação. Engenharia Agrícola, Jaboticabal, v. 26, n. 3, p. 695-703, 2006 a.

Metodologia para obtenção do hidrograma de escoamento superficial em encostas e canais. Parte II: modelo computacional e análise de sensibilidade. Engenharia Agrícola, Jaboticabal, v. 26, n. 3, p. 704-712, 2006b.

SIMÕES, W. L.; FIGUEIRÊDO, V. B.; SILVA, E. L. Uso do cilindro infiltrômetro único em diferentes solos. Engenharia Agrícola, Jaboticabal, v. 25, n. 2, p. 359-366, 2005.

WOOLHISER, D. A.; SMITH, R. E.; GOODRICH, D. C. KINEROS: a kinematic runoff and erosion model: documentation and user manual. Report ARS-77, United States Department of Agriculture, Agricultural Research Service, Washington, DC, USA, 1990. 130 p.
YOUNG, C. B.; McENROE, B. M.; ROME, A. C. Empirical determination of rational method runoff coefficients. Journal of Hydrologic Engineering, West Lafayette, v. 14, n. 12, p. 1283-1289, 2009.

ZANETTI, S. S.; OLIVEIRA, V. P. S.; SOUSA, E. F.; ALMEIDA, F. T.; SUGAWARA, M. T.; SILVA, J. M.

A. Modelagem hidrológica em microbacia hidrográfica Parte II: teste do modelo HidroBacia. Revista Brasileira de Engenharia Agrícola e Ambiental, Campina Grande, v. 13, n. 4, p. 435-442, 2009b.

ZANETTI, S. S.; SILVA, J. M. A.; SOUSA, E. F.; OLIVEIRA, V. P. S.; ALMEIDA, F. T. Modelagem hidrológica em microbacia hidrográfica Parte I: aprimoramento do modelo HidroBacia. Revista Brasileira de Engenharia Agrícola e Ambiental, Campina Grande, v. 13, n. 4, p. 429-434, 2009a.

ZONTA, J. H.; MARTINEZ, M. A.; PRUSKI, F. F.; SILVA, D. D.; MONTEBELLER, C. A. Adequação dos parâmetros do modelo de Green-Ampt-Mein-Larson em condições de campo. Revista Brasileira de Engenharia Agrícola e Ambiental, Campina Grande, v. 14, n. 10, p. 1021-1029, 2010. 
